\title{
Management of Stroke in a Coronary Artery Disease Patient after Major Colorectal Surgical Procedure
}

\author{
Authors \\ Karthik Prakasam ${ }^{1}$, Gowri Shankar Anjaneyan ${ }^{1}$, SR Karthik ${ }^{2}$, Satish Logidasan ${ }^{3}$, \\ Kanimozhi Rathinasamy ${ }^{4}$, Arulraj Panchatcharam ${ }^{5}$, Prasana Vadhanan ${ }^{6}$, Ambal S \\ Govt Stanley Medical College, TN- 600001, India \\ Corresponding Author \\ Satish Logidasan \\ Department of Anesthesiology, Govt Stanley Medical College, TN- 600001, India \\ Email: drsatishlogi@gmail.com
}

\begin{abstract}
The incidence of LV thrombus is approximately $40 \%$ following an Anterior Myocardial Infarction ${ }^{(1)}$. Embolism from thrombus is regarded as the most common cause of Stroke following MI with an incidence as high as $10 \%$ (prethrombolytic era) \& $2-3 \%$ (thrombolytic era) ${ }^{(2)}$. A patient with apical thrombus has a high risk of perioperative thromboembolism ${ }^{(3)}$, which could be avoided, to an extent with judicious use of anticoagulants.
\end{abstract}

Keywords: coronary artery disease, myocardial infarction, $l v$ thrombus.

\section{Perioperative Course}

39-year-old gentleman with complaints of bleeding per rectum was diagnosed as hyperplastic polyposis colon with infiltrating moderately differentiated adenocarcinoma of rectum was posted for Total proctocolectomy. He was a smoker \& alcoholic quitted 2years back when he developed Anterior wall MI (Not lysed) along with Inferior wall MI (Lysed). Post MI he was found to have a apical thrombus. He was started on Aspirin 150mg OD, Clopidogrel 75mg OD, Enalapril $2.5 \mathrm{mg}$ BD, Metoprolol 25mg BD, and Atorvostatin 40mg HS. His cardiac workup revealed Hypokinesia of Distal $2 / 3^{\text {rd }}$ of IVS/AW/AS wall of LV with a EF of $45 \%$ with moderate LV dysfunction \& LV Thrombus attached to Anteroapical part of LV $\left(18^{*} 11 \mathrm{~mm}\right)$ \& was not mobile. He was started on Inj Enoxaparin 40mg subcutaneous OD by the cardiologist. He was taken under high risk for General Anaesthesia. Pressures were monitored with Invasive Blood pressure monitoring. CVC cannulated \& supports kept ready. Intraoperative period was uneventful. MAP was maintained between $60-90 \mathrm{~mm} \mathrm{Hg}$. He was extubated \& shifted to Postoperative HDU. Six hours later patient suddenly became restless, threw generalized tonic clonic seizures lasting for 2 minutes and Injection Midazolam $2 \mathrm{mg}$ iv bolus along with Inj Phenytoin loading dose followed by infusion started. Half an hour later patient had another episode of seizures with partial limb movement appeared to be a complex partial seizure \& Injection Lorazepam $2 \mathrm{mg}$ iv given. His pressures fell to $100 / 50 \&$ he developed tachycardia of HR 120/min. He had bradypnea and started desaturating. 
He was intubated immediately with a sleeping dose of Thiopentone, paralysed with vecuronium and put on control mode. Midazolam sedation along with fentanyl infusion was given. His pressures were stabilized with Inj Dobutamine \& Inj Dopamine. Neurologist opinion obtained. He was started on Inj. Mannitol 100ml TDS, Inj Dexamethasone 6mg iv TDS. CT Brain after stabilization was adviced. He was extubated the next day after adequate recovery of consciousness \& BP stabilization. He was found to have Right Hemiparesis with UMN type of Right facial nerve palsy with Global Aphasia. He was shifted for CT Brain - infarct. He was started on Aspirin 150mg, Clopidogrel 75mg, Acitrom, Atorvostatin $80 \mathrm{mg}$ via ryles tube \& Inj Enoxaparin 60mg sc. Passive limb physiotherapy, routine bladder \& skin care given. Patient was shifted back to postoperative ward \& discharged later. He was adviced for neurology, cardiology, and surgical follow up at discharge.

\section{Discussion}

LV thrombus occurs in $7-46 \%$ in setting of Acute MI. Incidence is highest during first 3 months following Acute MI. ${ }^{(6)}$ In the recent years incidence of LV thrombus has decreased due to Smaller infarct, Remodelling of Heart, Use of Heparin. The Pathogenesis of LV thrombus mimics Virchow's triad. (7)

1. Blood stasis (LV regional wall Akinesia/ dyskinesia)

2. Endothelial Injury (Ischemia leads to subendocardial injury with inflammatory changes)

3. Hypercoagulability (ACS displays a hypercoagulable state - Increase concentration of Prothrombin, Fibrinopeptide A, vWF. Decrease concentration of enzyme responsible for clearing $\mathrm{vWF}$.

LV Thrombus is commonly seen in large anterior wall infarcts, Severe Apical a synergy, LV aneurysm, CHF (wherever there is worsening of LV systolic function) \& in use of Betablockers. But LV thrombus plays a positive role in acutely infarcted myocardium by offering mechanical support to the infracted myocardium \& protects against LV rupture. The thrombus becomes firmly attached to its site of origin, enhancing the underlying myocardial scar, limiting potential Infarct expansion $\&$ partially restoring the thickness of the myocardial wall. As a consequence bulging is reduced, resulting in a more effective myocardial contraction. "Delayed Enhancement Cardiac Magnetic Resonance Imaging (CMR) is the gold standard test for detecting LV Thrombus"

\section{Thromboembolism in LV Thrombus \& Neurological complications}

Factors that cause LV Thrombus to embolise are

1. Thrombus protruding into ventricular cavity

2. Exhibits Independent mobility

3. Severe CHF

4. Diffuse LV dilatation \& Systolic dysfunction

5. Previous Embolisation

6. Atrial Fibrillation

7. Advanced patient age

8. Doubtful factors like Thrombus size, Central echolucency, Hyperkinesia of the myocardial segments adjacent to the thrombus

Neurological factors to indicate an embolic mechanism:

1. Sudden onset

2. LOC at onset

3. Seizures at onset

4. Headache

5. Onset during an activity

6. Nausea/vomiting

7. Evidence of $>=2$ focal deficits

Prophylaxis of Perioperative Thromboembolism Thrombolysis

Nowadays antithrombotic therapy is thought to prevent embolic complications of LV thrombus.

Vaitkus and Barnathan collected

Datas from six studies comprising a total of 390 patients and assessed the incidence of LV thrombus formation in those patients treated with thrombolysis versus those without thrombolytic therapy. They were unable to demonstrate a statistical difference in the incidence of $\mathrm{LV}$ 
thrombus formation, only a trend in favour of thrombolysis $^{8}$

It was inferred that fibrinolytic agents are capable of lysing ventricular thrombi but that the risks of this therapy are too high.

\section{Role of Heparin}

In a study with 23 consecutive patients with mobile and protruding thrombi, high dose heparin was given intravenously over a period of 14-22 days. In all 23 patients LV thrombi decreased in size, with disappearance of the high risk features. No embolic events were detected during treatment, and the only complication was an upper gastrointestinal haemorrhage. Dalteparin, a low molecular weight heparin, reduced the incidence of LV mural thrombus formation but had no influence on the risk of systemic embolisation, and its use was associated with an increased risk of haemorrhage.

\section{Role of Vitamin K Antagonists (VKA)}

1. Observational studies conducted in the prethrombolytic and thrombolytic eras have provided support for the hypothesis that anticoagulation reduces the risk of embolisation

2. Both current European Society of Cardiology and American College of Cardiology/American Heart Association guidelines recommend vitamin $\mathrm{K}$ antagonist therapy in patients with an LV thrombus after myocardial infarction.

3. However, vitamin $\mathrm{K}$ antagonists do not appear to affect the likelihood of resolution of the thrombus and, unfortunately, no large randomised trials have been performed to evaluate the efficacy of long term anticoagulation to prevent embolisation in patients with LV thrombus. Therefore the effects of long-term anticoagulants on the risk of embolisation are the subject of debate. Withdrawal of anticoagulant medication has been proposed in long term since the risk of embolisation decreases over time, likely as a result of organisation of thrombus, which includes thrombus neovascularisation.

Retrospective studies documented ongoing embolic risk in LV thrombus patients. In indium-111 platelet imaging studies most thrombi, regardless of age, have been observed to have externally detectable ongoing platelet accumulation, indicating continued surface activity. The European guidelines recommend vitamin $\mathrm{K}$ antagonist for at least 3-6 months, while the American guidelines recommend indefinite treatment in patients without increased risk of bleeding.

4. There are limited data regarding the appropriate follow-up and timing of cessation of vitamin $\mathrm{K}$ antagonists in these patients, the following approach seems appropriate for most patients:

(i) Assess LV thrombus within the first month after AMI, preferably with CMR in high risk patients, and start vitamin $\mathrm{K}$ antagonist when $\mathrm{LV}$ thrombus is present and no contraindication exists

(ii)Re-evaluate LV thrombus formation after 6 months since data show that LV thrombus resolution in the initial months is very common, also in patients treated with vitamin $\mathrm{K}$ antagonists

5. When LV thrombus is not present and there is no other indication for vitamin $\mathrm{K}$ antagonist, assess bleeding risk and consider stopping therapy.

6. Newer anticoagulants are presently being developed and some of them are already registered. It can be envisioned that in the longer term these new anticoagulants will replace vitamin $\mathrm{K}$ antagonists. At present vitamin $\mathrm{K}$ antagonist therapy is still the standard of care for the treatment of $\mathrm{LV}$ thrombus. More importantly, the newer anticoagulants also have the risk of fatal and non-fatal bleedings and their role in LV thrombus patients should be further assessed.

7. Antiplatelet therapy and triple therapy in the PCI era 
8. Another issue is that nowadays STEMI patients are treated by primary PCI and receive long term dual antiplatelet therapy (including aspirin and a $\mathrm{P}_{2} \mathrm{Y}_{12}$ inhibitor). Consequently, patients with LV thrombus or at increased risk of LV thrombus after a myocardial infarction are frequently being treated with vitamin $\mathrm{K}$ antagonist in addition to dual antiplatelet therapy (triple antithrombotic therapy) and therefore are subjected to an increased bleeding risk. It is unclear, however, if long term anticoagulation is still necessary in STEMI patients treated by primary PCI and subsequent dual antiplatelet therapy.

9. Large prospective studies show a yearly incidence of bleeding of approximately $3.7 \%$ for dual antiplatelet therapy and $12 \%$ for triple antithrombotic therapy. The most common site of bleeding is the gastrointestinal tract $(30-40 \%)$ and cerebrum $(9-10 \%)$, with $25 \%$ of episodes in the latter site proving fatal. Non-fatal bleedings are an important predictor of mortality post-PCI at follow-up. Moreover, in the general STEMI population treated with primary PCI and dual antiplatelet therapy but no anticoagulation therapy, symptomatic cerebral infarction is rare, occurring in $0.75-1.2 \%$ of all STEMI patients. The potential benefit of vitamin $\mathrm{K}$ antagonist treatment on top of dual antiplatelet therapy may not outweigh the increased bleeding risk. This calls for a randomised trial to be conducted to determine whether anticoagulation treatment prevents embolic complications in AMI patients treated with primary PCI.

\section{Guidelines 2014 (AHA) Acute MI and LV Thrombus \\ Patients with large anterior MI associated with an LV ejection fraction $<40 \%$ and anteroapical wall motion abnormalities are at an increased risk of developing LV mural Thrombus (Stasis of blood in}

the ventricle and endocardial injury with associated inflammation)

No studies have examined the efficacy and safety of the newer antithrombotic agents (dabigitran, rivoraxaban, apixaban or fondaparinux) for prevention of LV thrombus or stroke in patients with acute MI.

MI and Thrombus Recommendations 2014 Recommendation Revisions (2011) Treatment with VKA therapy (target INR 2.5, range 2.0-3.0) for 3 months may be considered in patients with ischemic stroke or TIA in the setting of acute anterior STelevation MI without demonstrable left ventricular mural thrombus formation but with anterior apical akinesis or dyskinesis identified by echocardiography or other imaging modality. (Class IIb, LOE C) New Recommendation In patients with ischemic stroke or TIA in the setting of acute MI complicated by left ventricular mural thrombus formation and/or anterior or apical wall motion abnormalities with a left ventricular ejection fraction $<40 \%$ who are intolerant to VKA therapy due to non-hemorrhagic adverse events, treatment with LMWH, dabigatran, rivaroxaban, or apixaban for 3 months may be considered for prevention of recurrent stroke or TIA.

\section{Treatment of perioperative Thromboembolism}

Therapeutic anticoagulation is the standard treatment for thromboembolic disease and is suggested by the 2009 American College of Cardiology Foundation/American Heart Association guidelines for LV thrombus that has embolized However, guidelines regarding the management of $\mathrm{LV}$ thrombus are not definitive for cases in which the mass is $>5.0 \mathrm{~cm}$; but it is maintained that with therapeutic anticoagulation, the majority of $\mathrm{LV}$ thrombi will resolve without evidence of systemic embolization

Surgical thrombectomy is indicated in patients with recurrent emboli despite therapeutic anticoagulation and in patients with additional indications for cardiac surgery 


\section{Conclusion}

Despite anticoagulation at a therapeutic INR, our patient's thrombus embolized multiple times

This case supports the proclivity of massive, mobile thrombus to embolize and indicates the limitations of current management for this clinical challenge

\section{References}

1. Nayak D, Aronow WS, Sukhija R, McClung $\mathrm{JA}$, Monsen CE, et al. Comparison of frequency of left ventricular thrombi in patients with anterior wall versus nonanterior wall acute myocardial infarction treated with antithrombotic and antiplatelet therapy with or without coronary revascularization. Am J Cardiol. 2004; 93:1529-30. [PubMed]

2. Witt BJ, Ballman $\mathrm{KV}$, Brown $\mathrm{RD}$, Meverden RA, Jacobsen SJ, et al. The incidence of stroke after myocardial infarction: a meta-analysis. Am J Med. 2006; 119:354.e1-e9. [PubMed]

3. Dantzig JM, Delemarre BJ, Bot H, Visser CA. Left ventricular thrombus in acute myocardial infarction. Eur Heart J. 1996; 17:1640-5. [PubMed]

4. Asinger RW, Mikell FL, Elsperger J, et al Incidence of left-ventricular thrombosis after acute transmural myocardial infarction. Serial evaluation by two-dimensional echocardiography. N Engl J Med 1981; 305:297-302.

5. Domenicucci S, Bellotti P, Chiarella Fet al Spontaneous morphologic changes in left ventricular thrombi: a prospective twodimensional echocardiographic study. Clinical study with serial echocardiographic studies demonstrating spontaneous morphologic changes in LV thrombi.

6. Vaitkus PT, Barnathan ES. Embolic potential, prevention and management of muralthrombus complicating anteriormyocardial infarction: ameta-analysis. J Am Coll Cardiol (1993) 22(4): 10049.doi:10.1016/0735-1097(93)90409-T.
7. Mackman N. Triggers, targets and treatments for thrombosis. Nature 2008; 451:914-918.

8. Vaitkus PT, Barnathan ES. Embolic potential, prevention and management of mural thrombus complicating anterior myocardial infarction: a meta-analysis. J Am Cross Ref PubMed Web of Science Google Scholar. Relevant meta-analysis regarding the embolic potential and management of LV thrombus.) 